\title{
Parkinson's disease: if you can't change the outcome how can you qualify the journey?
}

\begin{abstract}
Parkinson's disease is the second most prevalent neurodegenerative disease in the world population. It is incurable and affects people of all ages. The progressive worsening of motor and non-motor symptoms of this disease makes its management complex in the medium and long term, demanding specialized interdisciplinary actions and focused on palliative care. Therefore, this mini review aims to describe the importance of palliative care in Parkinson's disease as a method of care to promote the quality of life to those involved in the journey in which its outcome is still inevitable.
\end{abstract}

Keywords: palliative, palliative care, parkinson parkinson's, disease, advanced stage, end of life, interdisciplinary, disease progression
Volume 2 Issue 2 - 2018

\author{
Michelle Hyczy de Siqueira Tosin,' Claudio \\ Antonio da Cruz Mecone, ${ }^{2}$ Beatriz Guitton \\ Renaud Batista de Oliveira ${ }^{3}$ \\ 'PhD student, Fluminense Federal University, Brazi \\ ${ }^{2}$ Specialization in Neuroscience Applied to Learning, Federal \\ University of Rio de Janeiro, Brazil \\ ${ }^{3}$ Department of Nursing Fundamentals and Administration, \\ Fluminense Federal University, Brazil
}

Correspondence: Michelle Hyczy de Siqueira Tosin, Estrada dos Bandeirantes 6953, Rio de Janeiro, RJ, Brazil, Tel +55 21 98842-0262, Email michellehyczy@gmail.com

Received: April 14, 2017 | Published: April 02, 2018

\section{Abbreviations: PD, parkinson's disease}

\section{Introduction}

"This is a personal story, sadly tragic and heart-breaking, but by sharing this information with you I know that you can help make a difference in the lives of others. (...) My hope is that it will help you understand your patients along with their spouses and caregivers a little more. And as for the research you do, perhaps this will add a few more faces behind the why you do what you do", said Mrs. Susan Schneider Williams at the special editorial of the Neurology journal, in 2016. ${ }^{1}$ The manuscript written not by a neuroscientist, but by a wife, who was complicit in the suffering caused by a neurodegenerative disease, enables us to understand the urgent need for the adequacy of therapeutic care. Her husband, the actor Robin Williams, was diagnosed with Parkinson's disease (PD), but three months after his death, the condition known as "Lewy Body Dementia" was confirmed by the results of necropsy, a condition included in the spectrum of parkinsonian diseases. This manuscript reveals the complexity of the facts experienced daily in clinics, doctors' office and hospitals, where entire families are faced with the diagnosis of an incurable disease. In this year, in which the PD commemorates the 200 years since the publication of the monograph of James Parkinson, ${ }^{2}$ many observations can be made from the narrative not only of Mrs. Susan Williams but of all the family members who are fighting against the biological clock. The untiring search for cure and treatment of the symptoms of this disease can distract health professionals from the daily reality experienced by patients and their families. ${ }^{3}$ False hopes can be unconsciously instilled and these will consequently produce suffering and frustration for all involved. For this reason, the shift from the health care paradigm to those suffering from a chronic and progressive neurological condition is essential for the quality of care. This change requires a look beyond cure, a look at palliative care.

Therefore, this mini review aims to describe the importance of palliative care in PD as a method of care to promote quality of life to those involved in the journey in which its outcome is still inevitable.

\section{Discussion}

Parkinson's disease is the second most common neurodegenerative disease in the world population, affecting 1 in 1000 individuals. ${ }^{4}$ It is clinically manifested by motor symptoms (rigidity od the muscles, tremor, postural instability and bradykinesia) and non-motor symptoms (bladder, bowel, sleep, mood, sensory and neuropsychiatric dysfunctions). ${ }^{5}$ Despite the exponential advance of scientific knowledge in the last two centuries, there is still no perspective of cure for this chronic neurological disease, which has an impact on the life of the patient and his family. ${ }^{6}$ Treatment of PD involves medications, neurosurgical procedures and non-drug therapies.

The drugs are intended to suppress motor and non-motor signs and symptoms of the disease. ${ }^{7}$ The neurosurgical procedure is indicated for patients who present a low response to antiparkinsonians. ${ }^{8}$ And nondrug therapy aims to promote patient safety, functionality and quality of life through physical therapy, occupational therapy, psychotherapy, among others. ${ }^{9}$ However, it is observed that these treatments promote the patient's well-being in the short and medium term, since in the long term the deleterious effects of the disease lead to the failure of all these therapies.

The degree of dissatisfaction and distress of the patient and their relatives increases with the impact of the misunderstood prognosis of this disease. This can be exemplified by the report of Mrs. Susan Williams regarding the presence of the gastrointestinal, urinary, sleep, anosmia and neuropsychiatric symptoms presented by her husband and that they did not correlate with the illness. ${ }^{1}$ The lack of knowledge about the disease and its progression left them confused, distressed, lost, impotent. After all, what is the expected reaction to the unknown? What are the attitudes that the patient and his family must take in the face of adversity? Is there any autonomy left to the patient / family to decide on the management of a progressive disease?

These and other questions can be better answered when the professional approach is based on palliative care, where patient / family engagement, through the exchange of knowledge, enables everyone to participate in decision making. For this reason, some 
scientists have been dedicated to studying palliative care in PD as a method of care to qualify the care provided to patients and their relatives / caregivers. ${ }^{10-14}$ The evolution of the concept of palliative care, which in the 1950s and 1960s was focused on pain relief and nutritional support to the patient at the end of life, made it possible to enlarge the professional approach in health in order to subsidize coping with problems related to the disease through the prevention and relief of suffering, early identification and accurate assessment for the treatment of pain and other physical, psychosocial and spiritual problems. ${ }^{14}$ Evidence also shows that chronic, sudden, incidental, or congenital chronic neurological diseases tend to evolve in a similar way to those affected by oncological diseases, leading to pain, respiratory failure, delirium, agitation, somnolence, nausea, vomiting and depression.

With regard to physical problems, in neurodegenerative diseases, palliative care aims to explore the patient's physical condition and, when this is no longer possible, to provide comfort. For psychosocial problems, palliative care subsidizes coping with the disease, minimizes social situations of stress and frustration, and also enables the family to organize itself financially and legally in the face of the imminent end of life. Under this approach, how different would the actions taken by the Williams family in the course of the disease? As clinicians and scientists, we express our gratitude for the opportunity to read an article written by a family that, in representing so many other families, makes us reflect on our behavior and, above all, makes us adjust our actions in favor of human life. After all, if the outcome is inevitable, we must qualify the journey!

\section{Conclusion}

The interdisciplinary care in chronic and incurable neurological conditions reveals a singular complexity, mainly because of the impact on the lives of individuals and their families in the short, medium and long term.

In patients with $\mathrm{PD}$, treatment also focused on the principles of palliative care is essential to qualify care, since functional and clinical impairment of the patient during the natural course of the disease requires dynamic adaptation of multidisciplinary health interventions.

\section{Acknowledgements}

None.

\section{Conflict of interest}

The authors declare that they have no potential conflicts of interest.

\section{References}

1. Williams SS. The terrorist inside my husband's brain. Neurology. 2016;87(13):1308-1311.

2. Obeso JA, Jon Stoessl A, Stamelou M. Editors' Note: The 200th anniversary of the shaking palsy. Mov Disord. 2017;32(1):1.

3. Miyasaki JM, Kluger B. Palliative care for Parkinson's disease: has the time come? Curr Neurol Neurosci Rep. 2015;15(5):26.

4. Lang AE, Shulman G. Perspectives parkinson's disease at 200 years: progress, new faces, and unmet needs. UTMJ. 2016;93(2):6-8.

5. Tosin MH de S, Oliveira BGRB de. The role of nurses in parkinson 's disease. Challenges in Parkinson's Disease. 2016;365-389.

6. Przedborski S. The two-century journey of Parkinson disease research. Nat Rev Neurosci. 2017;18(4):251-259.

7. Connolly BS, Lang AE. Pharmacological Treatment of Parkinson Disease. Jama. 2014;311(16):1670-1683.

8. De Rosa A, Tessitore A, Bilo L, et al. Infusion treatments and deep brain stimulation in Parkinson's Disease: The role of nursing. Geriatr Nurs. 2016;37(6):434-439.

9. Bloem BR, Vries NM, Ebersbach G. Nonpharmacological Treatments for Patients with Parkinson's Disease. Mov Disord. 2015; 30(11):1504-1520.

10. Miyasaki JM. Palliative care in Parkinson's disease. Curr Neurol Neurosci Rep. 2013;13(8):367.

11. Walker RW. Palliative care and end-of-life planning in Parkinson's disease. J Neural Transm. 2013;120(4):635-638.

12. Saleem TZ, Higginson IJ, Chaudhuri KR, et al. Symptom prevalence, severity and palliative care needs assessment using the Palliative Outcome Scale: a cross-sectional study of patients with Parkinson's disease and related neurological conditions. Palliat Med. 2013;27(8):722-731.

13. Richfield EW, Jones EJS, Alty JE. Palliative care for Parkinson's disease: a summary of the evidence and future directions. Palliat Med. 2013;27(9):805-810.

14. Ghoche R. The conceptual framework of palliative care applied to advanced Parkinson's disease. Parkinsonism Relat Disord. 2012;18(Suppl 3):S2-5. 